\title{
Quantifying Solid-State Mixtures of Crystalline Indomethacin by Raman Spectroscopy Comparison with Thermal Analysis
}

\author{
Eman Atef, ${ }^{1}$ Harsh Chauhan, ${ }^{2}$ Dev Prasad, ${ }^{1}$ Dunesh Kumari, ${ }^{3}$ and Charles Pidgeon' \\ ${ }^{1}$ Department of Pharmaceutical Sciences, School of Pharmacy, Massachusetts College of Pharmacy and Health Sciences, \\ 179 Longwood Avenue, Boston, MA 02115, USA \\ ${ }^{2}$ School of Pharmacy and Health Professions, Creighton University, Hixson-Lied Building. 152, 2500 California Plaza, Omaha, \\ NE 68178, USA \\ ${ }^{3}$ Department of Chemistry and Chemical Biology, Northeastern University, 414 Hurtig Hall, Boston, MA 02115, USA
}

Correspondence should be addressed to Eman Atef, eman.atef@mcphs.edu

Received 7 December 2011; Accepted 25 December 2011

Academic Editor: R. Nageswara Rao

Copyright (C) 2012 Eman Atef et al. This is an open access article distributed under the Creative Commons Attribution License, which permits unrestricted use, distribution, and reproduction in any medium, provided the original work is properly cited.

\begin{abstract}
This paper investigates Raman spectroscopy as a quick and reliable method to quantify the alpha $(\alpha)$ and gamma $(\gamma)$ polymorphic forms of indomethacin compared to differential scanning calorimetry (DSC). Binary mixtures with different ratios of $\alpha$ and $\gamma$ indomethacin were prepared and analyzed by Raman and DSC. The Raman method was found to be more reliable and superior compared to DSC. The partial conversion of the alpha to gamma polymorphic form during the DSC measurement was the major limitation for the use of full DSC as a quantitative method and resulted in difference between the calculated and measured enthalpy of both polymorphic forms.
\end{abstract}

\section{Introduction}

Polymorphism is a commonly encountered phenomenon in pharmaceutical formulations and final products. Scientists' interest in understanding pharmaceutically relevant aspects of polymorphism have increased over the years, in particular the drugs' different metastable polymorphic forms, the polymorphic forms dissolution rate, and their thermodynamic properties, as well as the in vivo drug kinetics [1-3].

The appearance or disappearance of a defined drug polymorphic form during manufacturing or storage is a major concern in drug process development [4-7]. Thus rapid and reliable quantification of polymorphic forms of active drug ingredients (API) is essential during drug manufacturing [8$10]$.

DSC monitors the heat changes associated with physical transformations; this includes transformation from (i) amorphous to crystalline form, (ii) crystalline drug to a different polymorph form, and (iii) crystalline drug to an amorphous solid-state mixture. Thus, DSC is routinely used to characterize amorphous systems, polymorphic changes studies, and in the quantification of the amorphous and crystalline contents in solid mixture [11-13]. The quantity of one polymorphic form can be estimated by either heat of crystallization or heat of fusion $[14,15]$. A major limitation in using DSC as a quantitative tool is the relative thermodynamic stability of the polymorphic forms. The interconversion of the polymorphic forms during the thermograms collection is the chief obstacle in the extensive use of DSC as a quantitative tool. Experimental modifications and validation may be required to overcome this potential problem.

IR has widely been used in quantitative and qualitative analysis of drugs in solid-state mixtures and blends. Although near infrared (IR) has been a dominating technique for the past decades, there is a significant increase in using Raman spectroscopy in solid- and liquid-state quantitative analysis [16]. Raman is complementary to IR and is used to identify and quantify different polymorphic forms $[17,18]$. Raman is a nondestructive method and requires minimal or no sample preparation. In addition, its low moisture interference is a major advantage over the IR spectroscopy. On the other hand, the Raman instruments are expensive, and small sampling area may result in misleading spectra depending on the used laser beam size [19]. 


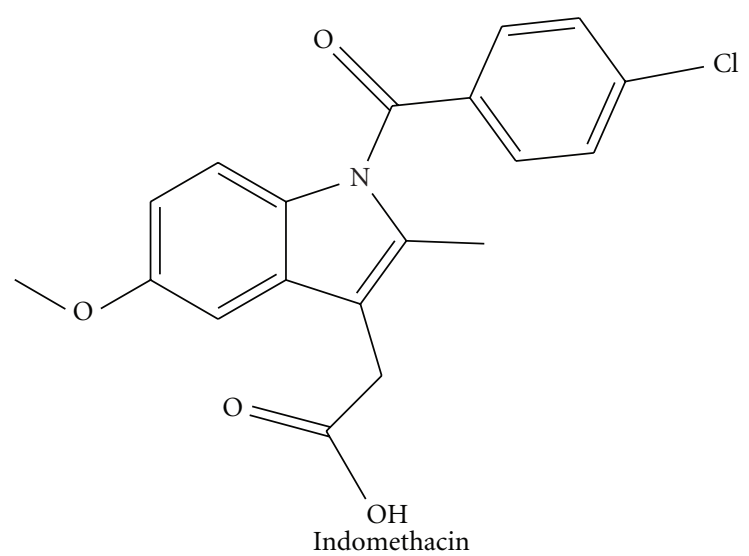

FIGURE 1: Indomethacin structure.

In our study we evaluated the Raman and DSC to quantify indomethacin polymorphic forms in solid-state mixtures.

Indomethacin (Figure 1) is a frequently used model drug in numerous studies including many in our laboratory. Being a poorly soluble, highly crystalline drug with two polymorphic forms, different approaches have been attempted to identify the factors affecting the drug crystallization kinetics in addition to investigating new techniques to improve its dissolution and interfere with its crystallization tendency. A key point to perform a successful crystallization or precipitation kinetics study that involves indomethacin different polymorphic forms is the development of a fast, reliable quantitative analysis method, which is the main goal of our current study.

In summary, we developed a simple, rapid, and reliable method to quantify a binary mixture of $\gamma$ and $\alpha$ indomethacin, which can be used to determine the relative amount of each polymorph when performing precipitation kinetics studies of indomethacin.

\section{Material and Methods}

2.1. Material. Indomethacin, $\gamma$ polymorphic form was purchased from Sigma Aldrich. The $\alpha$ indomethacin polymorph was prepared by dissolving $4 \mathrm{~g}$ of $\gamma$ indomethacin in $30 \mathrm{~mL}$ ethanol at $60^{\circ} \mathrm{C}$ to which $4-5 \mathrm{~mL}$ of distilled water was added to precipitate indomethacin as $\alpha$ polymorphic form. After vacuum filtration, the $\alpha$ indomethacin was dried overnight in a vacuum oven $[20,21]$.

2.2. Verification of the Indomethacin Reference Forms. The pure polymorphic forms were confirmed using DSC (SSC/5200 DSC 220, Seiko Instrument). The melting points of $152-154^{\circ} \mathrm{C}$ and $160-162^{\circ} \mathrm{C}$ corresponding to the $\alpha$ and $\gamma$, respectively, were used to verify the polymorphic forms. Raman spectroscopy (RXN1-785 Analyzer, Kaiser Optical Systems, Inc., Ann Arbor, Michigan) was used to confirm the DSC findings utilizing the Raman active vibrations in Table 1 .
2.3. Mixtures Preparation. Nine binary mixtures of $\alpha$ and $\gamma$ polymorphic forms were prepared using the following ratios $1: 9,2: 8,3: 7,4: 6,5: 5,6: 4,7: 3,8: 2$, and $9: 1$ of $\gamma$ to $\alpha$ forms. The 2 polymorphs were mixed using geometric dilution on a glass slide using a metal spatula.

2.4. Acquisition and Processing of Raman Spectra. The powder mixture samples were gently compressed using a glass slide to minimize the specular and diffuse reflectance and other sources of light scattering that decrease the detected Raman signals. Raman spectra were obtained using a microscope and Kaiser's HoloMap software. Data processing was performed using Grams AI version 8.0 software (Thermo, Inc.). The target surface area irradiated by the laser using a $5 \mathrm{x}$ objective was $\sim 80-100 \mu \mathrm{m}$. The optical bench was an RXN1-785 Analyzer equipped with an excitation laser operating at $785 \mathrm{~nm}$. The excitation cable was $64.5 \mu \mathrm{m}$ in diameter. Data acquisition used an accumulation time of 20 seconds, 3 accumulations, and a laser power setting of $400 \mathrm{~mW}$. The Raman data were exported into Grams AI 8.0 data processing software. The peak intensity of $\alpha$ indomethacin at $\bar{\nu} 1632 \mathrm{~cm}^{-1}$ and $\gamma$ indomethacin at $\bar{v} 1700 \mathrm{~cm}^{-1}$ was obtained after baseline correction from $1732 \mathrm{~cm}^{-1}$ to $1665 \mathrm{~cm}^{-1}$ and from $1665 \mathrm{~cm}^{-1}$ to $1632 \mathrm{~cm}^{-1}$, respectively. Peak positions varied by less than $0.3 \mathrm{~cm}^{-1}$. Three spectra were collected for each sample, and the peak intensities were averaged and used for data analysis. Each concentration was run as triplicate.

2.5. DSC Experimental Details. Calorimetric analysis was carried out using SSC/5200 DSC 220, Seiko Instruments. Samples between (5-10 mg) were placed in a closed DSC aluminum pan and heated at a constant rate of $10^{\circ} \mathrm{C} / \mathrm{min}$ until $200^{\circ} \mathrm{C}$. These experiments were performed in a dry nitrogen environment. High-purity tin and indium were used for calibration before the experiments. Experiments were performed in triplicate and the mean values were calculated. The data was analyzed and processed using EXSTAR software. The $\Delta \mathrm{H}$ of fusion were measured for both pure $\alpha$ and $\gamma$ forms of indomethacin.

The observed values of $\alpha$ (M.P. $152-154^{\circ} \mathrm{C}, \Delta \mathrm{H}$ fusion $97 \mathrm{~mJ} / \mathrm{mg}$ ) and $\gamma$ (M.P. $160-162^{\circ} \mathrm{C}, \Delta \mathrm{H}$ fusion $106 \mathrm{~mJ} / \mathrm{mg}$ ) were similar to the reported values and were used in our study for the quantitative calculations [22].

The measured enthalpy was obtained directly from the melting endotherms of physical mixtures of $\alpha$ and $\gamma$ polymorphs. It was plotted against the polymorphic form percentage in the mixture. The theoretical enthalpy of physical mixtures was calculated based on the contribution of each polymorphic form assuming they are in a mixture of inert substance. The enthalpy of each individual polymorph was calculated based on its percentage in the tested powder blend.

The individual theoretical enthalpy of each polymorph was calculated based on the percentage of each polymorphic form in the tested mixtures and the enthalpy of the pure polymorph. 
TABLE 1: The alpha and gamma indomethacin-specific Raman and IR peaks and their bond assignments used in our study [21].

\begin{tabular}{lcc}
\hline Polymorphic form & Raman peak $\left(\mathrm{cm}^{-1}\right) /$ peak assignment & IR peak $\left(\mathrm{cm}^{-1}\right) /$ peak assignment \\
\hline Alpha & 1648 hydrogen bonded acid $(\mathrm{C}=\mathrm{O})$ stretch & 1649 hydrogen bonded acid $(\mathrm{C}=\mathrm{O})$ \\
& 1680 hydrogen bonded acid $(\mathrm{C}=\mathrm{O})$ & 1681 hydrogen bonded acid $(\mathrm{C}=\mathrm{O})$ \\
1692 benzoyl $(\mathrm{C}=\mathrm{O})$ & $\begin{array}{c}1688 \text { benzoyl }(\mathrm{C}=\mathrm{O}) \\
\text { Gamma }\end{array}$ & 1733 nonhydrogen bonded acid $(\mathrm{C}=\mathrm{O})$ \\
\hline \multirow{2}{*}{1698, Benzoyl $(\mathrm{C}=\mathrm{O})$} & 1717 asymmetric acid $(\mathrm{C}=\mathrm{O})$ of a cyclic dimer \\
1698 benzoyl $(\mathrm{C}=\mathrm{O})$
\end{tabular}

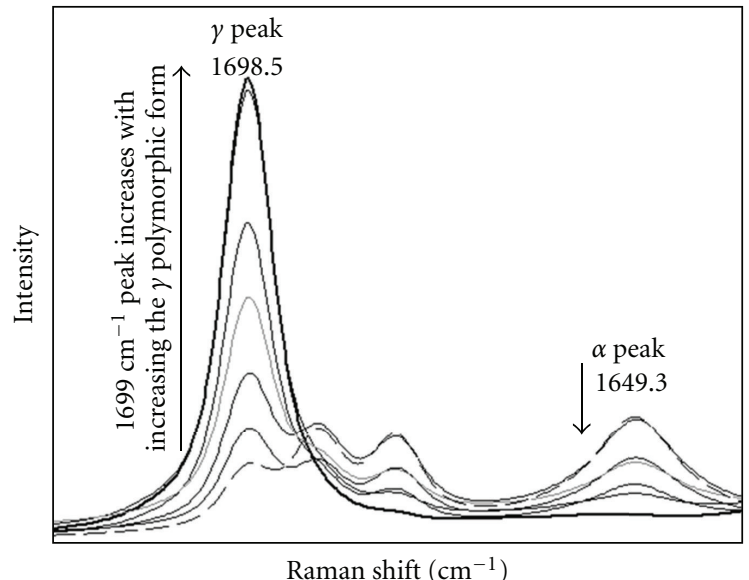

FIGURE 2: Raman spectra of mixtures of different $\alpha$ and $\gamma$ indomethacin ratios between $1600-1800 \mathrm{~cm}^{-1}$ shows an increase in the $1699 \mathrm{~cm}^{-1}$ peak with increasing the $\gamma$ indomethacin concentration in the mixture. The spectra are for different $\gamma: \alpha$ ratios; the top bold spectrum corresponds to $\gamma: \alpha 9: 1$ and the bottom dashed corresponds to the $1: 9$ ratios.

\section{Results}

3.1. Verification of the Indomethacin Polymorphic Forms. The polymorphic forms of pure $\alpha$ and $\gamma$ indomethacin samples were confirmed using both Raman and DSC. The Raman peaks at $1699 \mathrm{~cm}^{-1}$ and $1648 \mathrm{~cm}^{-1}$ were used as reference peaks for the $\gamma$ and $\alpha$ forms, respectively [21]. Two endothermic peaks were observed in the DSC thermograms. The first corresponded to $\alpha$ indomethacin at $151-154^{\circ} \mathrm{C}$ with enthalpy of $106 \mathrm{~mJ} / \mathrm{mg}$ and the second corresponded to the pure $\gamma$ form at $159-162^{\circ} \mathrm{C}$ with enthalpy of $97 \mathrm{~mJ} / \mathrm{mg}$. The DSC melting point and enthalpy of fusion were of agreement of the previously published values for both the $\gamma$ and $\alpha$ indomethacin [22].

\subsection{Vibrational Spectroscopy}

3.2.1. Raman. In order to quantify the $\alpha$ and $\gamma$ indomethacin mixtures, we utilized the carbonyl group region (Figure 2 ). The studied peaks are described in Table 1. The ratio of the peak intensity of $I \bar{\nu}_{1699} \mathrm{~cm}^{-1} / I \bar{\nu}_{1648} \mathrm{~cm}^{-1}$ was plotted against the $\alpha$ form weight fraction in the generated mixtures. The data showed a good fit on the logarithmic scale (Figure 3), $\left(R^{2}=0.94\right)$.

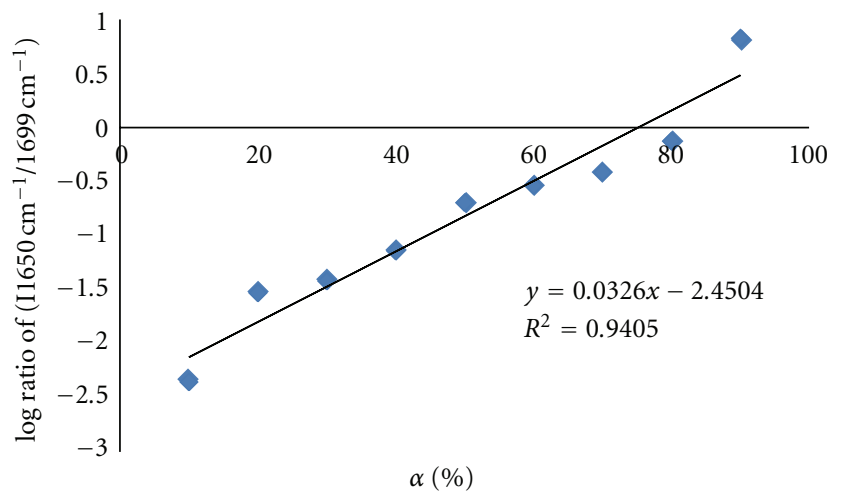

FIgURE 3: The intensity ratio of the $I_{1650} \mathrm{~cm}^{-1} / I 1699 \mathrm{~cm}^{-1}$ as a function of weight percentage of the $\alpha$ indomethacin in generated mixtures of different $\alpha$ and $\gamma$ ratios.

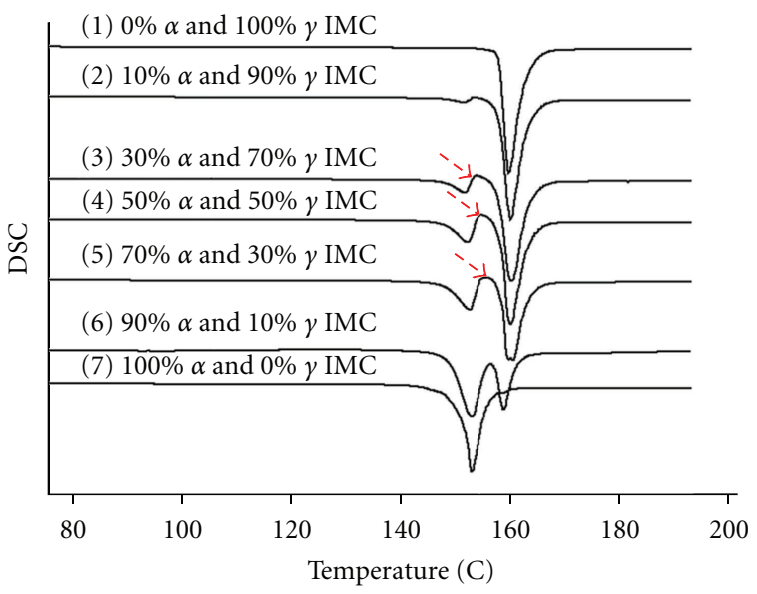

FIGURE 4: DSC thermograms of different $\alpha-\gamma$ polymorphic forms mixtures showing the clear recrystallization of the $\alpha$ in some of these mixtures (3-5).

3.2.2. DSC. The melting endotherms of different ratio mixtures of the $\alpha$ and $\gamma$ indomethacin forms are shown in Figure 4. The endotherms were used to calculate the melting enthalpy of $\alpha$ and $\gamma$ polymorphic forms. Figure 4 also highlights the recrystallization of the $\alpha$ indomethacin during the thermograms collection. The measured and theoretical enthalpy of the $\alpha$ and $\gamma$ indomethacin are plotted against the corresponding polymorphic form concentration in the prepared mixtures in Figures 5 and 6, respectively. 


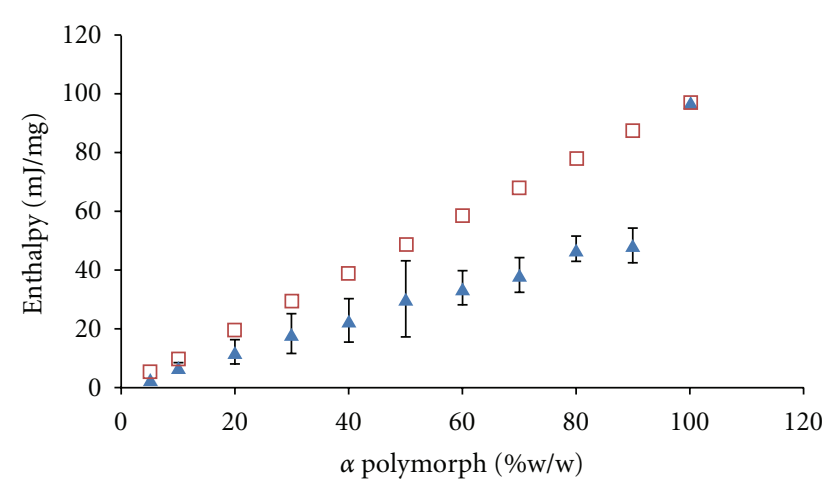

^ Measured enthalpy

$\square$ Theoretical enthalpy

Figure 5: The measured and theoretical enthalpies of the $\alpha$ indomethacin polymorph in different $\alpha-\gamma$ mixture ratios.

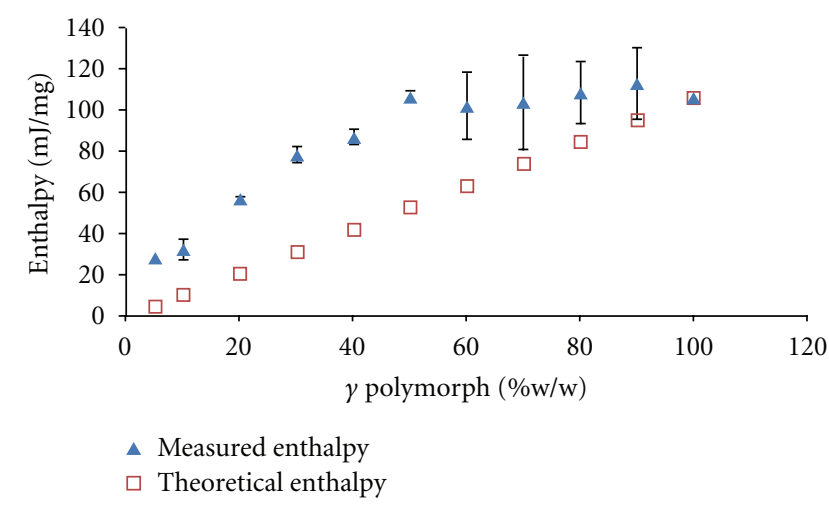

FIgURE 6: The measured and theoretical enthalpies of the gamma indomethacin polymorph in different $\alpha-\gamma$ mixture ratios showing initial straight line correlation followed by a plateau after $1: 1 \alpha-\gamma$ mixture.

Although the enthalpy of the $\alpha$ polymorphic form showed linear correlation with its concentration $\left(R^{2}=\right.$ 0.994), all measured values were lower than the calculated value, suggesting recrystallization of the $\alpha$ to the $\gamma$ form during the DSC thermograms collection. Consequently the gamma measured enthalpy values were higher than their corresponding calculated values as anticipated. The enthalpy of the gamma indomethacin showed linear correlation with its corresponding concentration up to the $50 \%$ gamma total concentration level, above which complete conversion of the $\alpha$ form was observed and confirmed by the plateau of the enthalpy-concentration curve.

\section{Discussion}

The use of spectroscopic techniques, especially the Raman spectroscopy, in quantitative analysis had significantly increased over the past few years. Raman has an increasing potential use in solid dosage form analysis and process analytical technology (PAT) applications [23].
As indicated earlier, the indomethacin crystal form can be identified by the Raman peak position of the reference spectra of the drug product. Raman vibrational spectroscopy is sensitive to any small bond environmental changes, and thus can be useful in distinguishing most crystal polymorphic forms. A major Raman spectroscopy advantage over IR is that due to the minor contribution of overtone vibrations in Raman spectra, the Raman peaks are sharp, wellresolved bands, compared to IR spectra [24]. Raman is also a quantitative spectroscopy method and has many applications as solid dosage forms quantitative analysis techniques. For example, quantifying the active ingredient in a final formulation, the tablet hardness determination [25] and tablet surface adsorbed moisture analysis [26].

The developed method is fast, simple, and accurate. The method is based on the relative intensity of $\alpha$ and $\gamma$ specific peaks. Both are sharp and nonoverlapping peaks. Such a reliable method is needed in the precipitation kinetics studies, where identifying the precipitates, their forms, and ratios is crucial in understanding the factors influencing the precipitation of a poorly soluble drug like indomethacin.

Using the DSC thermal analysis method in the quantification of the less stable $\alpha$ form of indomethacin was feasible using the change in enthalpy with the percentage weight of the $\alpha$ form in the tested mixture. On the other hand the quantification of gamma indomethacin was unachievable due to the recrystallization of the $\alpha$ form to the more stable $\gamma$ form, which explains the straight line single slope correlation between the enthalpy of fusion of the $\alpha$ form versus its percentage weight, compared to the changing slope observed with the corresponding $\gamma$ curve.

DSC is generally used as a qualitative tool for drug polymorphism studies. There are some challenges that hinder the extensive use of DSC in quantitative analysis such as (i) exhibition of multiple thermal events, (ii) recrystallization to metastable polymorphic forms, and (iii) broadening of solid melting peaks associated with this technique. Nevertheless, since it has been successfully used for the quantification of some amorphous/crystalline forms of some molecules, we wanted to explore its usefulness in the quantification of different indomethacin polymorphic forms using heat of fusion. We found DSC use in quantifying the indomethacin polymorphic forms to be limited and requires expensive experimental conditions adjustment and complicated calculations, mainly due to the conversion of the metastable form. On the other hand, at low concentration of one form (between 10-5\%), differentiating and quantifying the 2 polymorph was visible using DSC but not Raman.

A key factor, to keep in mind while considering the DSC for quantitative analysis is the stability of the polymorphic form throughout the experiment. One technique of minimizing the recrystallization of the low melting point polymorph is to run a high-speed differential scanning calorimetry (Hyper-DSC), a modified DSC method using accelerated heating rate $\left(200^{\circ} \mathrm{C} / \mathrm{min}\right.$ to $\left.400^{\circ} \mathrm{C} / \mathrm{min}\right)$ to inhibit recrystallization $[27,28]$.

The advantage of Raman spectroscopy over DSC in polymorphic form quantification is the ability to avoid samples heating, which accelerates the polymorphic form conversion. 
The used laser with low power and for very limited time minimizes the heat exposure.

It is important to clarify that the conclusions driven in this research, although they may apply to similar molecules, are specific to indomethacin.

\section{Conclusion}

We are reporting a method to quantify alpha $(\alpha)$ and gamma $(\gamma)$ indomethacin using Raman, and we are also reporting the difficulty involved in using DSC as a qualitative tool. The difference in the Raman spectral vibrations was successfully utilized to quantify the two indomethacin polymorphic forms in binary mixtures.

\section{Acknowledgment}

The authors thank Northeastern University Massachusetts College of Pharmacy and Health Sciences for providing the instruments used in this research.

\section{References}

[1] H. G. Brittain, "Polymorphism and solvatomorphism 2005," Journal of Pharmaceutical Sciences, vol. 96, no. 4, pp. 705-728, 2007.

[2] R. W. Lancaster, P. G. Karamertzanis, A. T. Hulme, D. A. Tocher, T. C. Lewis, and S. L. Price, "The polymorphism of progesterone: stabilization of a "disappearing" polymorph by co-crystallization," Journal of Pharmaceutical Sciences, vol. 96, no. 12, pp. 3419-3431, 2007.

[3] J. Bauer, S. Spanton, R. Henry et al., "Ritonavir: an extraordinary example of conformational polymorphism," Pharmaceutical Research, vol. 18, no. 6, pp. 859-866, 2001.

[4] J. E. Patterson, M. B. James, A. H. Forster, R. W. Lancaster, J. M. Butler, and T. Rades, "The influence of thermal and mechanical preparative techniques on the amorphous state of four poorly soluble compounds," Journal of Pharmaceutical Sciences, vol. 94, no. 9, pp. 1998-2012, 2005.

[5] A. K. Tiwary, "Modification of crystal habit and its role in dosage form performance," Drug Development and Industrial Pharmacy, vol. 27, no. 7, pp. 699-709, 2001.

[6] N. Sandler, J. Rantanen, J. Heinämäki, M. Römer, M. Marvola, and J. Yliruusi, "Pellet manufacturing by extrusion-spheronization using process analytical technology," AAPS PharmSciTech, vol. 6, no. 2, article 26, pp. E174-E183, 2005.

[7] S. Airaksinen, M. Karjalainen, E. Räsänen, J. Rantanen, and J. Yliruusi, "Comparison of the effects of two drying methods on polymorphism of theophylline," International Journal of Pharmaceutics, vol. 276, no. 1-2, pp. 129-141, 2004.

[8] M. E. Auer, U. J. Griesser, and J. Sawatzki, "Qualitative and quantitative study of polymorphic forms in drug formulations by near infrared FT-Raman spectroscopy," Journal of Molecular Structure, vol. 661-662, no. 1-3, pp. 307-317, 2003.

[9] K. Pöllänen, A. Häkkinen, S. Reinikainen et al., "IR spectroscopy together with multivariate data analysis as a process analytical tool for in-line monitoring of crystallization process and solid-state analysis of crystalline product," Journal of Pharmaceutical and Biomedical Analysis, vol. 38, no. 2, pp. 275-284, 2005.
[10] M. Trifkovic, S. Rohani, and M. Mirmehrabi, "Polymorphic generation through solvent selection: ranitidine hydrochloride," Organic Process Research and Development, vol. 11, no. 1, pp. 138-143, 2007.

[11] R. Lefort, A. De Gusseme, J. F. Willart, F. Danède, and M. Descamps, "Solid state NMR and DSC methods for quantifying the amorphous content in solid dosage forms: an application to ball-milling of trehalose," International Journal of Pharmaceutics, vol. 280, no. 1-2, pp. 209-219, 2004.

[12] H. Ahmed, G. Buckton, and D. A. Rawlins, "The use of isothermal microcalorimetry in the study of small degrees of amorphous content of a hydrophobic powder," International Journal of Pharmaceutics, vol. 130, no. 2, pp. 195-201, 1996.

[13] Z. Német, G. C. Kis, G. Pokol, and A. Demeter, "Quantitative determination of famotidine polymorphs: X-ray powder diffractometric and Raman spectrometric study," Journal of Pharmaceutical and Biomedical Analysis, vol. 49, no. 2, pp. 338-346, 2009.

[14] E. M. Phillips, "An approach to estimate the amorphous content of pharmaceutical powders using calorimetry with no calibration standards," International Journal of Pharmaceutics, vol. 149, no. 2, pp. 267-271, 1997.

[15] B. Shah, V. K. Kakumanu, and A. K. Bansal, "Analytical techniques for quantification of amorphous/crystalline phases in pharmaceutical solids," Journal of Pharmaceutical Sciences, vol. 95, no. 8, pp. 1641-1665, 2006.

[16] A. Heinz, M. Savolainen, T. Rades, and C. J. Strachan, "Quantifying ternary mixtures of different solid-state forms of indomethacin by Raman and near-infrared spectroscopy," European Journal of Pharmaceutical Sciences, vol. 32, no. 3, pp. 182192, 2007.

[17] Y. Hu, H. Wikström, S. R. Byrn, and L. S. Taylor, "Analysis of the effect of particle size on polymorphic quantitation by Raman spectroscopy," Applied Spectroscopy, vol. 60, no. 9, pp. 977-984, 2006.

[18] A. L. Enculescu and J. R. Steiginga, "Raman spectroscopya powerful tool for non-routine analysis of pharmaceuticals," American Pharmaceutical Review, vol. 5, no. 1, pp. 81-88, 2002.

[19] A. W. Newman and S. R. Byrn, "Solid-state analysis of the active pharmaceutical ingredient in drug products," Drug Discovery Today, vol. 8, no. 19, pp. 898-905, 2003.

[20] N. Kaneniwa, M. Otsuka, and T. Hayashi, "Physicochemical characterization of indomethacin polymorphs and the transformation kinetics in ethanol," Chemical and Pharmaceutical Bulletin, vol. 33, no. 8, pp. 3447-3455, 1985.

[21] L. S. Taylor and G. Zografi, "Spectroscopic characterization of interactions between PVP and indomethacin in amorphous molecular dispersions," Pharmaceutical Research, vol. 14, no. 12, pp. 1691-1698, 1997.

[22] X. Pan, T. Julian, and L. Augsburger, "Increasing the dissolution rate of a low-solubility drug through a crystalline-amorphous transition: a case study with indomethicin," Drug Development and Industrial Pharmacy, vol. 34, no. 2, pp. 221231, 2008.

[23] J. Johansson, S. Pettersson, and S. Folestad, "Characterization of different laser irradiation methods for quantitative Raman tablet assessment," Journal of Pharmaceutical and Biomedical Analysis, vol. 39, no. 3-4, pp. 510-516, 2005.

[24] M. E. Auer, U. J. Griesser, and J. Sawatzki, "Qualitative and quantitative study of polymorphic forms in drug formulations by near infrared FT-Raman spectroscopy," Journal of Molecular Structure, vol. 661-662, no. 1-3, pp. 307-317, 2003. 
[25] S. Virtanen, O. Antikainen, and J. Yliruusi, "Determination of the crushing strength of intact tablets using Raman spectroscopy," International Journal of Pharmaceutics, vol. 360, no. 1-2, pp. 40-46, 2008.

[26] E. Atef, H. Chauhan, M. Ceresia, and C. Pidgeon, "Using Raman spectroscopy in tablet moisture surface analysis: tablet surface markers," Journal of Pharmaceutical and Biomedical Analysis, vol. 53, no. 4, pp. 852-859, 2010.

[27] C. McGregor and E. Bines, "The use of high-speed differential scanning calorimetry (Hyper-DSC) in the study of pharmaceutical polymorphs," International Journal of Pharmaceutics, vol. 350, no. 1-2, pp. 48-52, 2008.

[28] C. McGregor, M. H. Saunders, G. Buckton, and R. D. Saklatvala, "The use of high-speed differential scanning calorimetry (Hyper-DSC) to study the thermal properties of carbamazepine polymorphs," Thermochimica Acta, vol. 417, no. 2, pp. 231-237, 2004. 


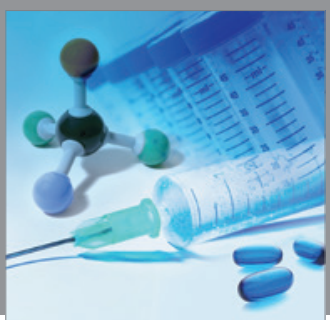

International Journal of

Medicinal Chemistry

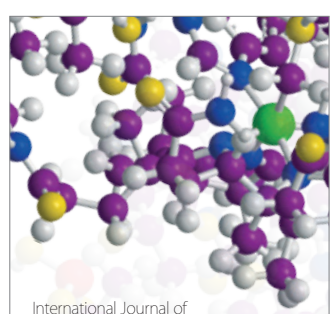

Carbohydrate Chemistry

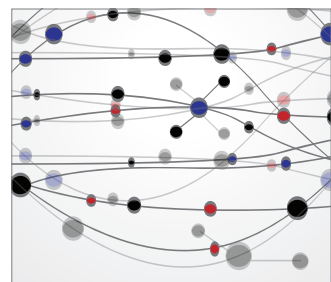

The Scientific World Journal
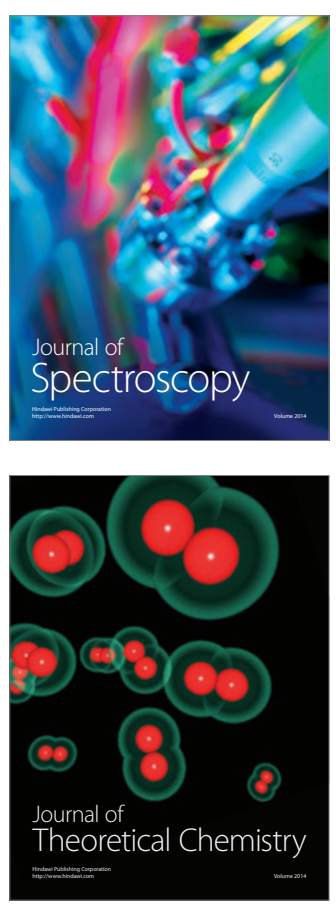
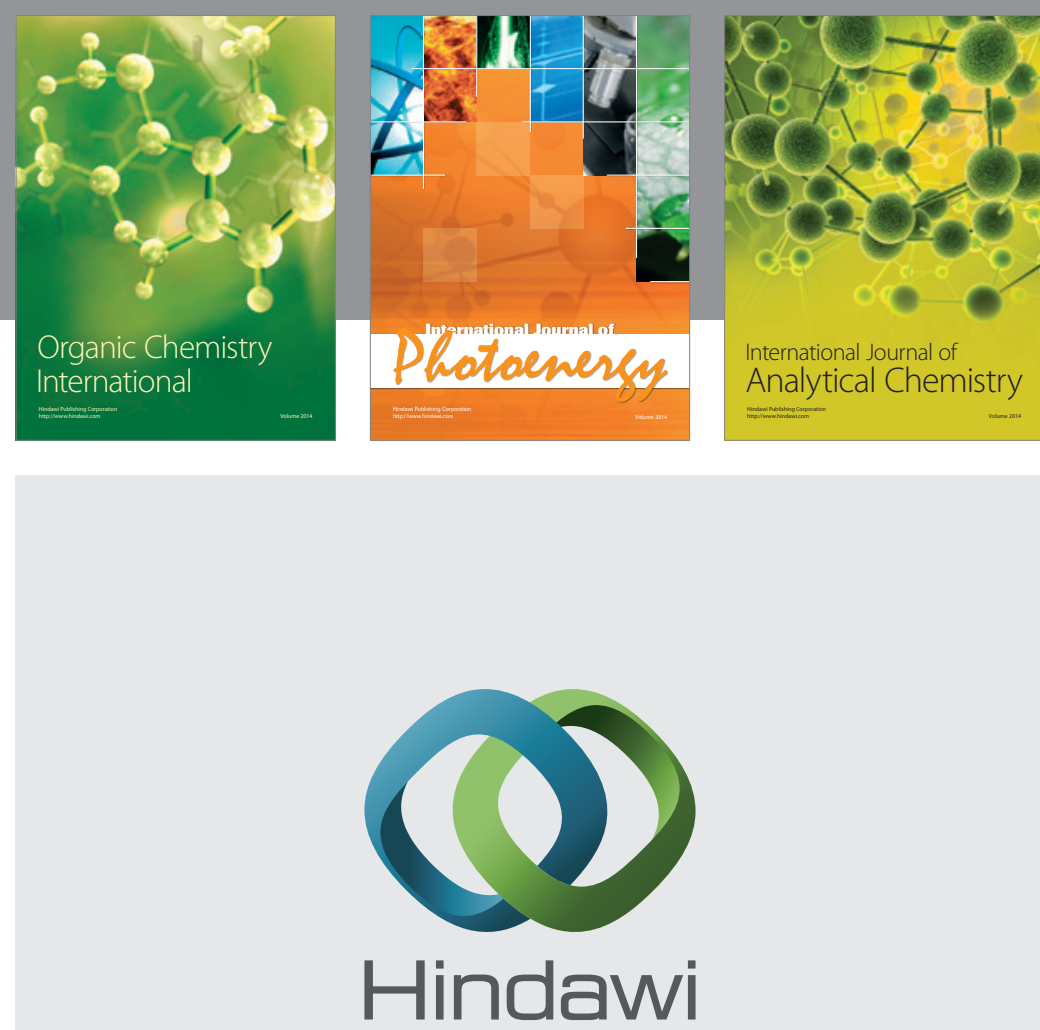

Submit your manuscripts at

http://www.hindawi.com
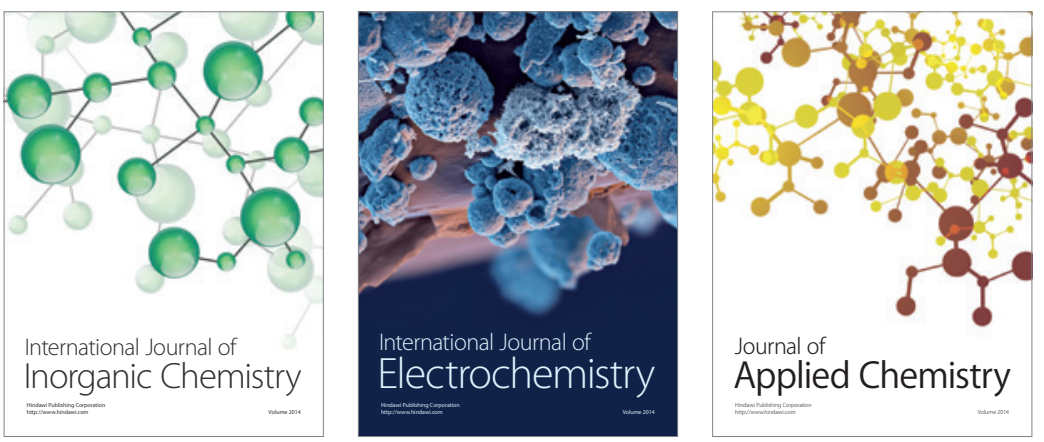

Journal of

Applied Chemistry
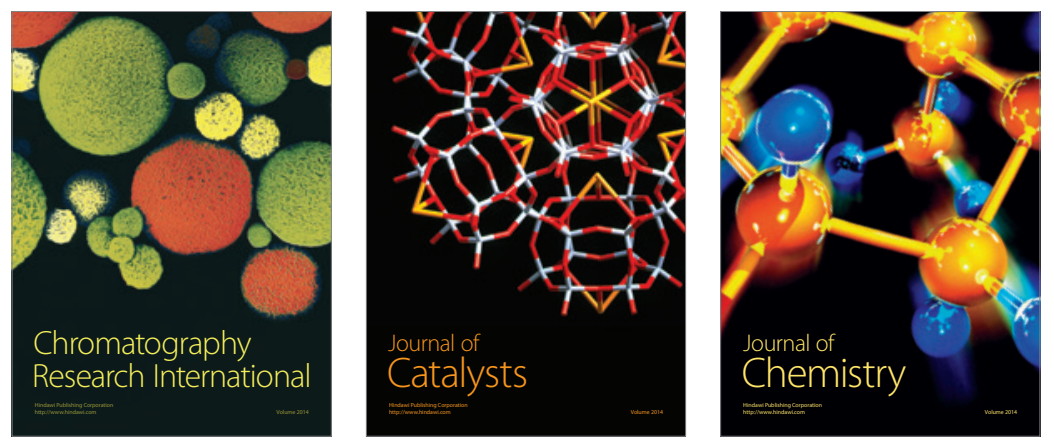
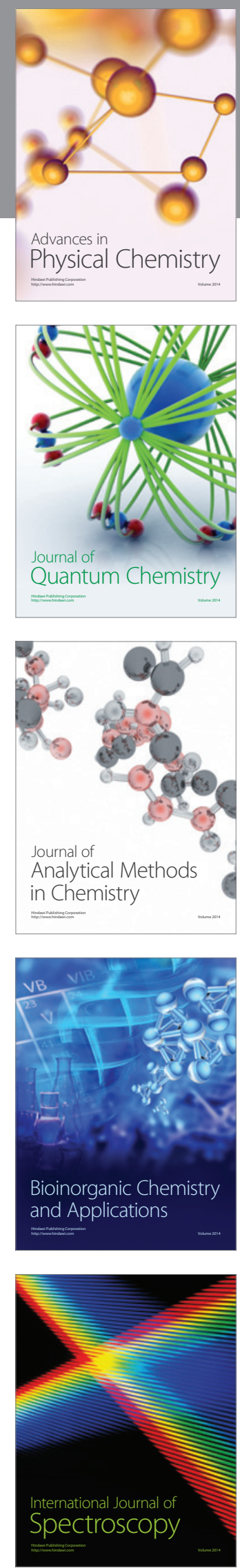